\title{
Engagement of vimentin intermediate filaments in hypotonic stress
}

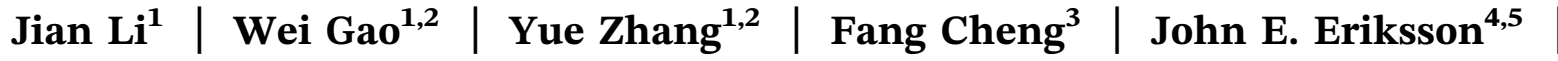 \\ Sandrine Etienne-Manneville ${ }^{6}$ | Yaming Jiu ${ }^{1,2}{ }^{10}$
}

${ }^{1}$ CAS Key Laboratory of Molecular Virology and Immunology, Institut Pasteur of Shanghai, Chinese Academy of Sciences, Shanghai, China

${ }^{2}$ University of Chinese Academy of Sciences, Beijing, China

${ }^{3}$ School of Pharmaceutical Sciences (Shenzhen), SYSU, China

${ }^{4}$ Cell Biology, Biosciences, Faculty of Science and Engineering, Åbo Akademi University, Turku, Finland

${ }^{5}$ Turku Centre for Biotechnology, University of Turku and Åbo Akademi University, Turku, Finland

${ }^{6}$ Institut Pasteur Paris CNRS UMR3691, Cell Polarity, Migration and Cancer Unit, Equipe Labellisée Ligue Contre le Cancer, Paris, France

\section{Correspondence}

Yaming Jiu, CAS Key Laboratory of Molecular Virology and Immunology, Institut Pasteur of Shanghai, Chinese Academy of Sciences, Shanghai, China.

Email: ymjiu@ips.ac.cn

\section{Funding information}

"100 talents program" from the Chinese Academy of Sciences; Key Laboratory of Molecular Virology \& Immunology, Grant/Award Number: KLMVI-OP201904; Institut Pasteur of Shanghai, Grant/Award Number: 1185170000

\begin{abstract}
Intermediate filaments (IFs) play a key role in the control of cell structure and morphology, cell mechano-responses, migration, proliferation, and apoptosis. However, the mechanisms regulating IFs organization in motile adhesive cells under certain physical/pathological conditions remain to be fully understood. In this study, we found hypo-osmotic-induced stress results in a dramatic but reversible rearrangement of the IF network. Vimentin and nestin IFs are partially depolymerized as they are redistributed throughout the cell cytoplasm after hypo-osmotic shock. This spreading of the IFs requires an intact microtubule network and the motor protein associated transportation. Both nocodazole treatment and depletion of kinesin-1 (KIF5B) block the hypoosmotic shock-induced rearrangement of IFs showing that the dynamic behavior of IFs largely depends on microtubules and kinesin-dependent transport. Moreover, we show that cell survival rates are dramatically decreased in response to hypo-osmotic shock, which was more severe by vimentin IFs depletion, indicating its contribution to osmotic endurance. Collectively, these results reveal a critical role of vimentin IFs under hypotonic stress and provide evidence that IFs are important for the defense mechanisms during the osmotic challenge.
\end{abstract}

\section{K E Y W O R D S}

cytoplasmic redistribution, hypo-osmosis, intermediate filaments, osmotic stress, vimentin

\section{1 | INTRODUCTION}

Cells are frequently submitted to stresses, such as mechanical stretching, changes in osmotic pressure, and chemically-induced stresses. Among these, the osmotic stress is a potent regulator of cells exposed to osmotically active environments, such as the musculoskeletal system, the kidney, the cardiovascular system, and the digestive

Abbreviations: IFs, intermediate filaments; U2OS cells, human osteosarcoma cells; DMEM, Dulbecco modified Eagle medium.

Li, Gao, and Zhang have contribute equally to the work. 
track. ${ }^{1-3}$ Hypo-osmotic shock is a common physiological stress that renal epithelial cells, articular chondrocytes, and pleural mesothelial cells can encounter.

Cell morphology, architecture, and ion-channel based transduction pathway are direct and pronounced physical effects upon hypo-osmotic stress. Besides, hypo-osmotic stress also promotes nuclear swelling limited by the stretch of the nuclear lamina and induces a smooth, round shape of the nucleus. ${ }^{4}$ It has been shown that actin filaments contribute to transcription regulation during osmotic shock associated with GEF-H1/RhoA/ROK/ MRTF signaling pathway. ${ }^{5}$ Astrocytes devoid of glial fibrillary acidic protein (GFAP) intermediate filaments (IFs) have a less effective response to osmotic stress measured by taurine efflux. ${ }^{6}$ Keratin IFs have been involved in keratinocyte recovery after hypo-osmotic shock. ${ }^{7}$ Alteration of the keratin network by dominantnegative mutations renders cells more sensitive to osmotic stress. ${ }^{6}$ Besides, D'Alesandro et $\mathrm{al}^{7}$ found that there is no fragmentation or disassembly of keratin detected during hypo-osmotic shock and the recovery period. ${ }^{6}$ However, whether and how hypo-osmotic challenge may affect other IF organization to provide mechanical and functional integrity of cells remain unraveled.

In this study, we witness that exposure to hypoosmotic stress causes a rapid cytoplasmic extension of both vimentin and nestin IFs. The normal perinuclear organization of the vimentin network is recovered when cells return to the iso-osmotic condition. Monitoring the early response to hypo-osmotic shock by immune staining, solubility test, and live-cell imaging revealed that vimentin filaments were partially disassembled during the reorganization. Moreover, the vimentin redistribution upon hypotonicity depends on microtubule integrity and its motor protein kinesin-1. Furthermore, depletion of vimentin IFs led cells more sensitive to hypo-osmotic stress-induced cell apoptosis. Collectively, our study demonstrates that hypo-osmosis stress leads to dramatic vimentin IFs reorganization which participates in cellular integrity and function.

\section{2 | MATERIALS AND METHODS}

\subsection{Cell culture and transfections}

Human osteosarcoma (U2OS) cells and HeLa cells were maintained in high glucose $(4.5 \mathrm{~g} / \mathrm{L})$ Dulbecco modified Eagle medium (DMEM) (BE12-614F, Lonza, Basel, Switzerland) supplemented with $10 \%$ fetal bovine serum (10500-064, Gibco, Waltham, MA), $10 \mathrm{U} / \mathrm{mL}$ penicillin,
$10 \mu \mathrm{g} / \mathrm{mL}$ streptomycin and $20 \mathrm{mM}$ L-glutamine (from 100X concentrate; Gibco) at $37^{\circ} \mathrm{C}$ in humidified atmosphere with $5 \% \mathrm{CO}_{2}$. Transient transfections were performed with FuGENE HD (Promega, Madison, WI) according to the manufacturer's instructions. Small interfering RNA (siRNA) experiments were performed with Lipofectamine RNAiMAX (Invitrogen) using $40 \mathrm{nM}$ On-target siRNA pool of KIF5B (Dharmacon, Lafayette, CO; target sequence 5'-AAAUAAG ACUCUACGGAAC- $3^{\prime}, \quad$ 5'-CCGCAAACGCUAUCAGCA A-3', 5'-CGUCCAAGCCUUAUGCAUU-3', 5'-CUGUGUCA ACGUAGAGUUA-3') or $40 \mathrm{nM}$ AllStars negative control siRNA (Qiagen, Hilden, Germany).

\section{2 | Plasmids and hypo-osmotic shock}

Vimentin-EGFP, vimentin-mCherry, nestin-GFP, and tubulin-GFP were kind gifts from John Eriksson (Turku, Finland). The hypo-osmotic medium was made by using complete growth medium diluted appropriately in deionized water (dilution 1:9 to obtain $30 \mathrm{mOsm}, 1: 4$ to obtain $60 \mathrm{mOsm}$, and 1:1 to obtain $150 \mathrm{mOsm}$ ) and used in all the experiments except Figure S1C. Alternatively, dialyzed serum was added into diluted DMEM with deionized water in Figure S1C which was used to verify the phenotype. Microtubule assembly was inhibited by $10 \mu \mathrm{M}$ nocodazole for 1.5 hours (\#2190S, Cell Signaling, Danvers, MA). Dynein was inhibited by $20 \mu \mathrm{M}$ ciliobrevin D for 45 minutes (\#M60041; Xcessbio, San Diego, CA) under serum deprivation to facilitate its efficient inhibition. Actin assembly was inhibited by $5 \mu \mathrm{g} / \mathrm{mL}$ cytochalasin D for 1 hour (\#PHZ1063; Thermo Fisher Scientific, Waltham, MA).

\subsection{Immunofluorescence and live-cell imaging}

Immunofluorescence experiments were performed as previously described. ${ }^{11}$ Cells were plated on CYTOOchips before fixation as previously described. ${ }^{11}$ The following primary antibodies were used: vimentin rabbit polyclonal antibody (dilution 1:100; Cell Signaling); F-actin was visualized with Alexa Fluor 488-, 568-, or 647- conjugated to phalloidin (dilution 1:200; Invitrogen, Carlsbad, CA). Secondary antibodies were conjugated to Alexa Fluor 488, 568, or 647 (Invitrogen). The time-lapse images were acquired with the 3I Marianas imaging system (3I intelligent Imaging Innovations, Denver, CO). After transient transfection, the cells were incubated for 24 hours and replated before imaging on $10 \mu \mathrm{g} / \mathrm{mL}$ fibronectin-coated glass-bottomed dishes (MatTek Corporation, Ashland, MA). 


\subsection{Analysis of the ratios of soluble to insoluble protein in cells}

The amounts of soluble and insoluble vimentin were evaluated by the method described previously. ${ }^{8,9}$ Cells were washed with ice-cold PBS and then collected by centrifugation at $8000 \mathrm{~g}$ for 5 minutes. The resulting pellets were incubated at $37^{\circ} \mathrm{C}$ for 30 minutes in buffer containing $1 \%$ Nonidet P-40, 10\% (v/v) glycerol, 20 mM N'-a-hydroxythylpiperazine-N'- ethanesulfanic acid (HEPES) (pH 7.4), $150 \mathrm{mM} \mathrm{NaCl}, 2 \mathrm{mM}$ sodium orthovanadate, $2 \mathrm{mM}$ molybdate, $2 \mathrm{mM}$ sodium pyrophosphate, and protease inhibitors. The soluble (disassembled) and insoluble (assembled) fractions were collected after centrifugation at $2100 \mathrm{~g}$ for 30 minutes at $4^{\circ} \mathrm{C}$ and assessed by immunoblot analysis using anti-vimentin antibody.

\section{5 | Statistics}

Statistics were performed with Excel (Microsoft, Redmond, WA) and SigmaPlot (SYSTAT Software Inc, Chicago, IL). For data following a normal distribution, the Student two-sample unpaired $t$ test was used. If data did not follow normal distribution, Mann-Whitney $U$ test for two independent samples was conducted.

\subsection{Ethical statement}

The study is not required ethical statement because there are no human participants or samples used in this study.

\section{3 | RESULTS}

\section{1 | Hypo-osmotic shock results in dramatical extension of IFs through cell cytoplasm}

To investigate the impact of hypo-osmotic stress on the IF network organization, we submitted cells to the hypoosmosis culture medium as previously described. ${ }^{10}$ In isoosmotic conditions, human osteosarcoma U2OS cells display an endogenous vimentin IFs mainly concentrated in the perinuclear region with only sparse filaments radiating toward the cell periphery. While under hypo-osmotic conditions for 10 minutes, both vimentin and nestin IFs spread homogeneously throughout the entire cell cytoplasm (Figure 1A and 1B). HeLa cell line was also tested which showed a similar temporal- and spatial-redistribution of vimentin IFs (Figure 1C). To allow more precise analysis, cells were plated on crossbow shaped micropatterns (Figure 1D; Figure S1A). By segmenting the cells, we quantified the distribution, which further confirmed the extension of vimentin IFs up under hypo-osmotic shock (Figure S1B).
In addition, there are increased protrusions and cytoplasmic curved actin fibers appeared upon hypo-osmotic shock (Figure S1A). Moreover, immunofluorescence showed that both vimentin and nestin IFs started extending towards the cell periphery as soon as 5 minutes and keep extended while under hypo-osmotic stress (Figure 1E; Figure S2A).

To explore the possibility that the rearrangements of vimentin IFs were due to the decreased concentration of nutrients, we used the dialysis method so that the serum concentration remained similar to that of the regular medium. The initiation and extension process of vimentin IFs was also observed upon hypo-osmotic stress with normal serum culture (Figure S1C). Collectively, these experiments reveal that hypo-osmotic stress triggers changes of cell morphology together with a profound rearrangement of vimentin and nestin IF network.

\section{2 | The extended vimentin IFs are partially disassembled under hypo-osmotic stress, and reversible when back in iso-osmotic culture}

Both vimentin and nestin appeared to change from filamentous to thin, short segments or squiggles. The solubility was thus tested and quantified (Figure 2A and 2B), which demonstrated that vimentin network underwent a process of depolymerization and repolymerization during hypo- and back isotonic condition.

Transient transfection of vimentin-EGFP and -mCherry induced the expression of the tagged protein to a level corresponding to approximately $10 \%$ of endogenous vimentin (measured by Western blot analysis with a transfection rate of 50\% in Figure S2B). We estimated that labeled proteins did not affect the organization of the endogenous vimentin. Live-cell imaging together with the corresponding kymograph and line profiles showed the rearrangement of the vimentin and nestin IFs (Figure 2C and 2D; Figure S2A and S2D). Interesting to note that microtubules appeared intact at least in the initial 5 minutes and further disturbed upon hypo-osmotic shock (Figure 2E). Moreover, we did not detect any sustain changes in the total amount of endogenous vimentin by Western blot (Figure S2E), suggesting that vimentin network redistribution may result from the movement of preexisting proteins rather than newly synthesis.

The normal perinuclear accumulation of vimentin can be recovered by exposing cells back to isotonic culture condition (Figure $2 \mathrm{~F}$ ), indicating that vimentin rearrangements under hypo-osmotic stress are reversible. In addition, actin stress fibers appear to spatially restrain the extension of vimentin IFs (yellow arrows in Figure 1E and Figure 2C), supporting previous studies that actin 


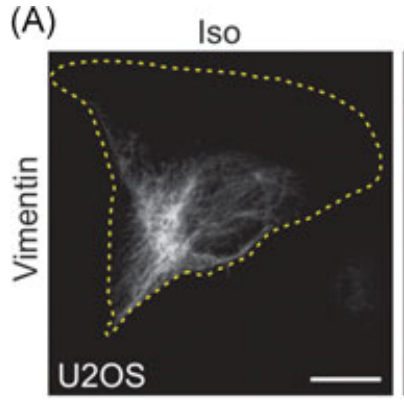

(C)

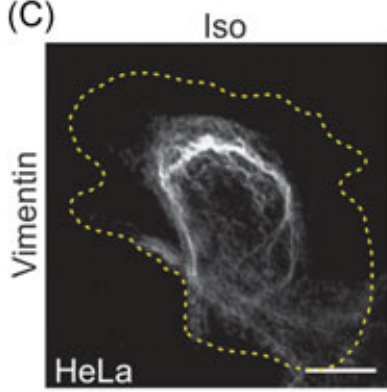

(E)

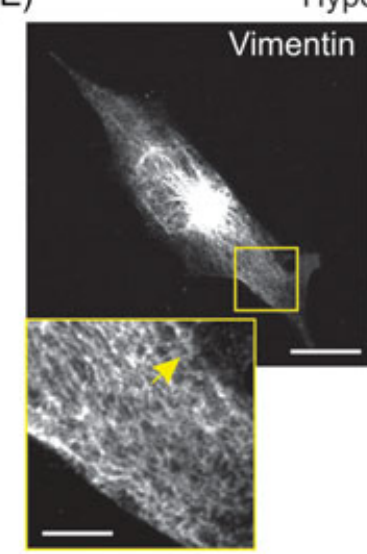

Hypo

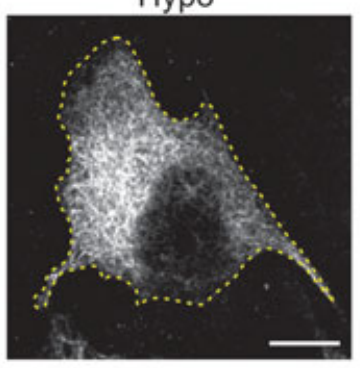

Hypo

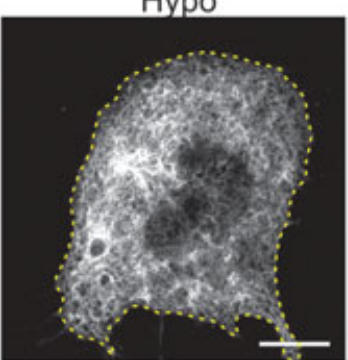

Hypo for 5'

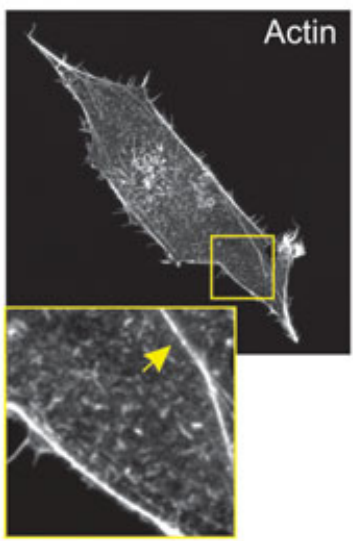

(B)

Iso

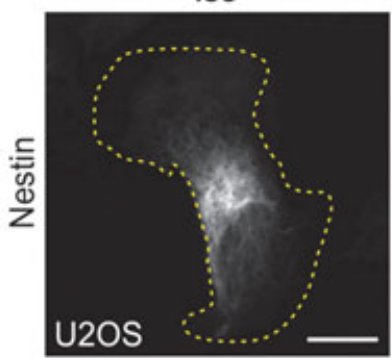

(D)
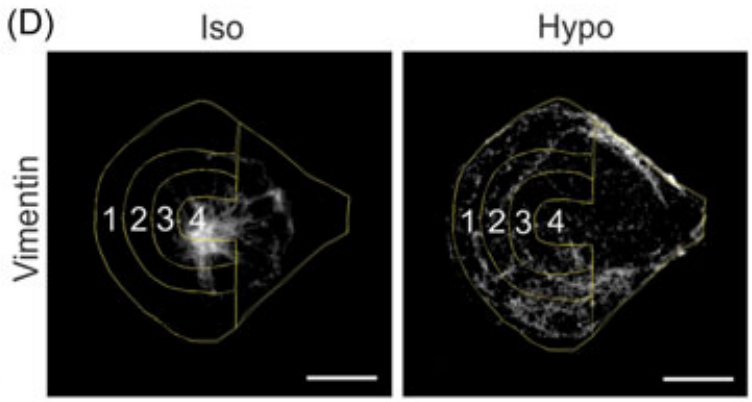

Hypo for 30'

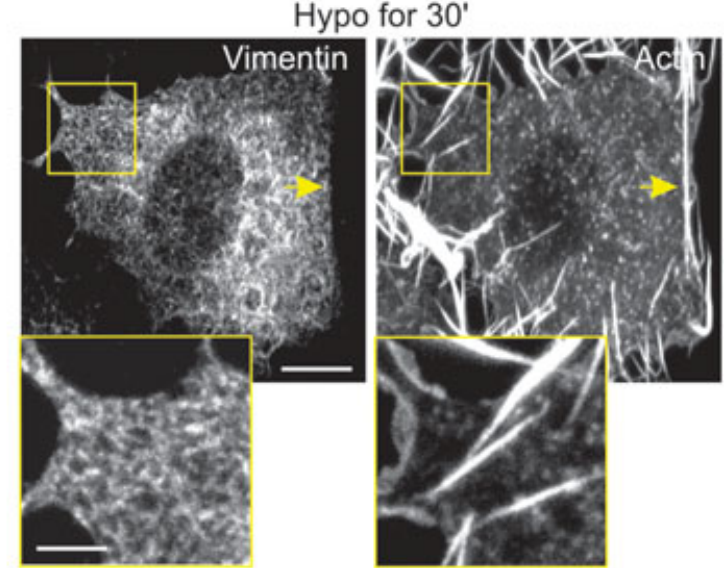

F I G U RE 1 IFs extend through cytoplasm upon hypo-osmotic shock. A,C, Endogenous vimentin IFs in both U2OS (A) and HeLa (C) cells extended under hypotonic stress. Bars $=10 \mu \mathrm{m}$. B, Endogenous nestin in U2OS cells extended under hypotonic stress. Bars $=10 \mu \mathrm{m}$. Yellow dash line indicated the outline of the cell. D, Localization of vimentin in iso- and hypotonic-treated cells grown on micropatterns. Bars $=10 \mu \mathrm{m}$. E, Immunofluorescence microscopy shows the extension of endogenous vimentin in 5 and 10 minutes upon hypotonic stress. Yellow arrows indicate that actin filaments play as a boundary during vimentin extension. Bars $=10 \mu \mathrm{m}$ (in cell images) and $2 \mu \mathrm{m}$ (in the magnified images). IF, intermediate filament

filaments associate with IFs to regulate its cytoplasmic localization. ${ }^{11-14}$

\section{3 | The extension of vimentin upon hypo-osmotic shock depend on microtubules and kinesin-dependent transport}

IFs rearrangements have been shown to rely on actindriven retrograde flow, and microtubule-dependent transport . ${ }^{1115-17}$ To determine the role of microtubules in IFs spreading, we used nocodazole to depolymerize microtubules, which totally blocked the extension of vimentin in hypo-osmotic conditions (Figure 3A and $3 \mathrm{~B})$. We next knocked down microtubule motor protein KIF5B by siRNAs (Figure S3A and S3B) which resembled the blockage of the vimentin network extension upon hypo-osmotic shock as in microtubule disruption condition (Figure 3A and 3B). In contrast, inhibition of another microtubule motor protein dynein and actin fibers, with ciliobrevin D and cytochalasin D, respectively, showed no apparent effects on hypoosmotic shock-induced vimentin IFs extension (Figure $3 \mathrm{~A}$ and 3B; Figure S3C). The effects were further verified and quantified when cells were plated on micropatterns (Figure 3C and 3D). 
(A)

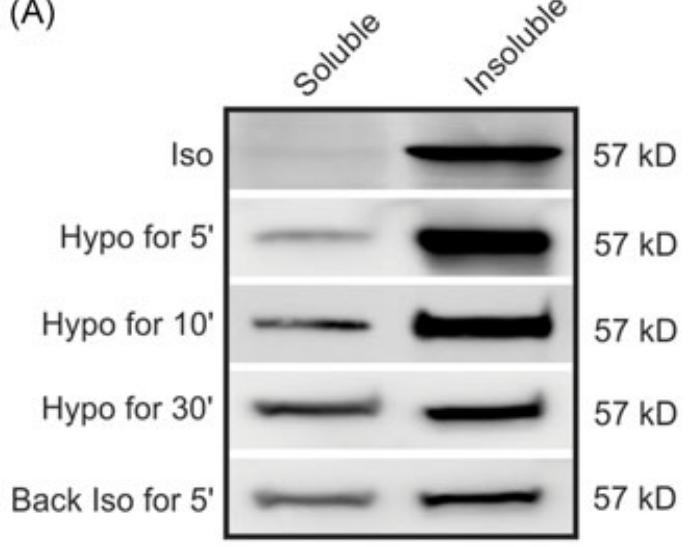

(B)

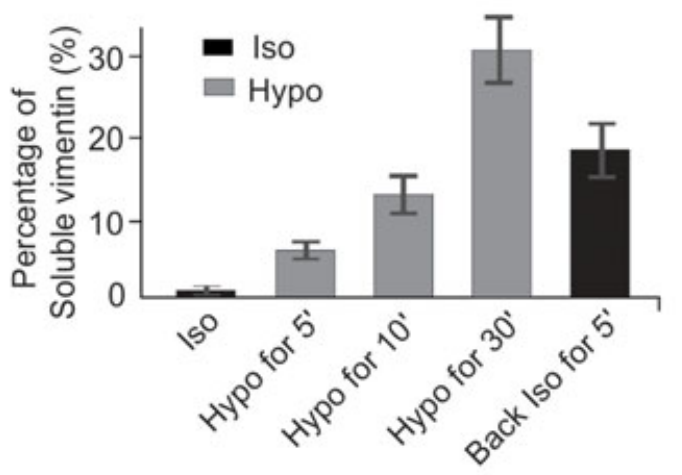

(C)
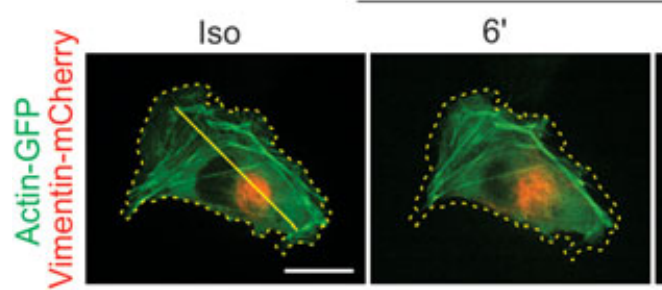

Hypo for

(D) Vimentin-mCherry

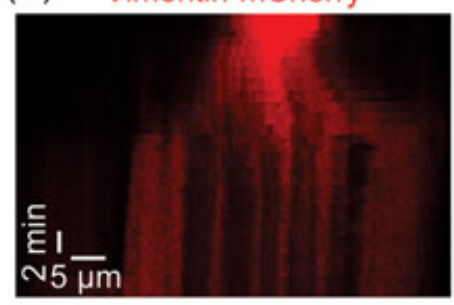

(F)

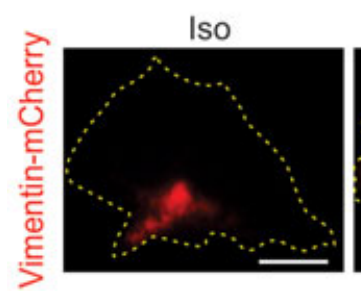

(E)

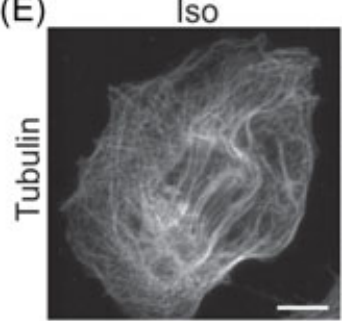

$10^{\prime}$
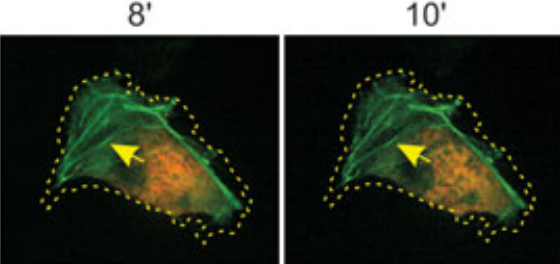

Hypo for $5^{\prime}$

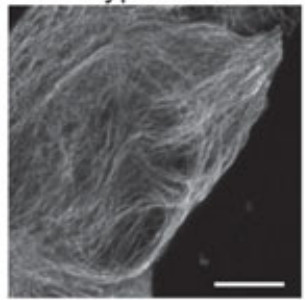

Iso for $1^{\prime}$

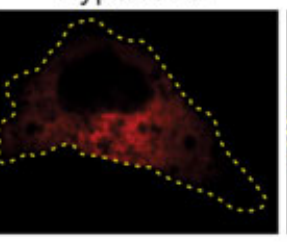

$3^{\prime}$

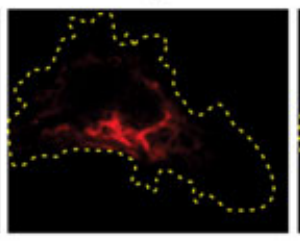

$18^{\prime}$

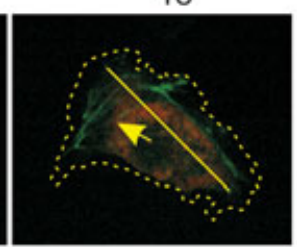

Hypo for $15^{\prime}$

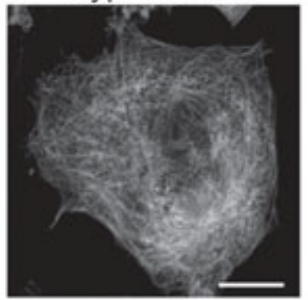

FIG U RE 2 Ectopic expressed vimentin IFs extend through cytoplasm upon hypo-osmotic shock. A,B, Western blot analysis and quantification of soluble/insoluble vimentin levels upon hypo-osmotic stress and back to isotonic normal culture. The data are presented as mean \pm SEM. Three independent experiments were analyzed. C, Time-lapse imaging of U2OS cells expressing actin-GFP and vimentin-mCherry resemble the extension as endogenous vimentin upon hypo-osmotic shock. Bars $=10 \mu \mathrm{m}$. D, Kymograph showed the vimentin fluorescence intensity profile along the yellow line in panel A over time. E, Immunofluorescence microscopy demonstrate that endogenous tubulin stays intact in the initial 5 minutes of hypo-osmotic shock. Bars $=10 \mu \mathrm{m}$. F, Time-lapse imaging of U2OS cells expressing vimentin-mCherry revealing that vimentin retracted when cell back to isotonic normal culture. Bars $=10 \mu \mathrm{m}$. Yellow dash line indicated the outline of the cell. IF, intermediate filament

Interestingly, vimentin IFs could also be extended when cells were grown on a softer substrate $(25 \mathrm{kPa})$ upon hypo-osmotic shock (Figure S4A), indicating that the microtubules and motor kinesin driven rearrangements of IFs upon osmotic shock are not sensitive to the substrate rigidity.

\subsection{Vimentin IFs function as a protective role during hypo-osmotic stress-induced apoptosis}

To investigate the possibility that vimentin IFs are involved in cell responses to hypo-osmotic shock, we 


\section{6 - WI LEY- Journal of Cellular Bioshemistry}

(A)
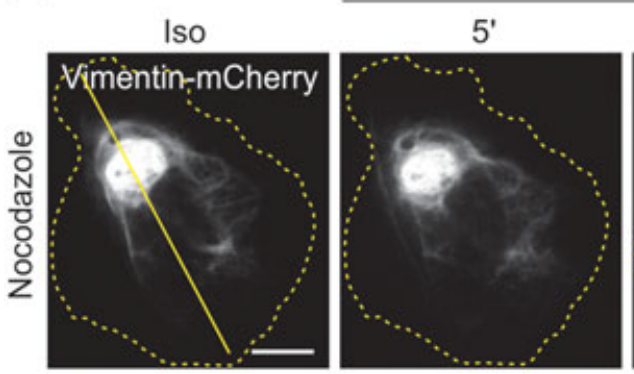

Hypo for
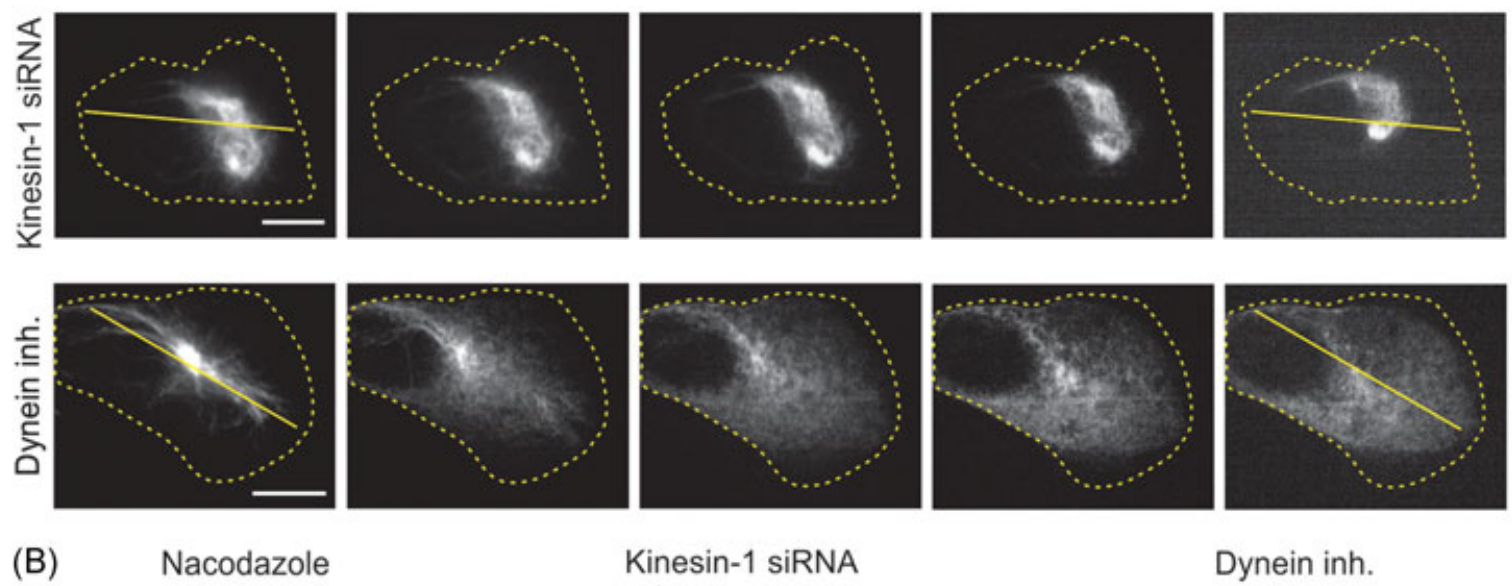

Kinesin-1 siRNA

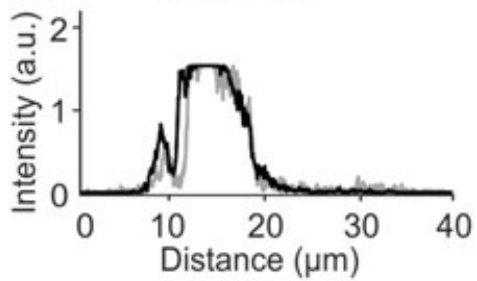

(C)
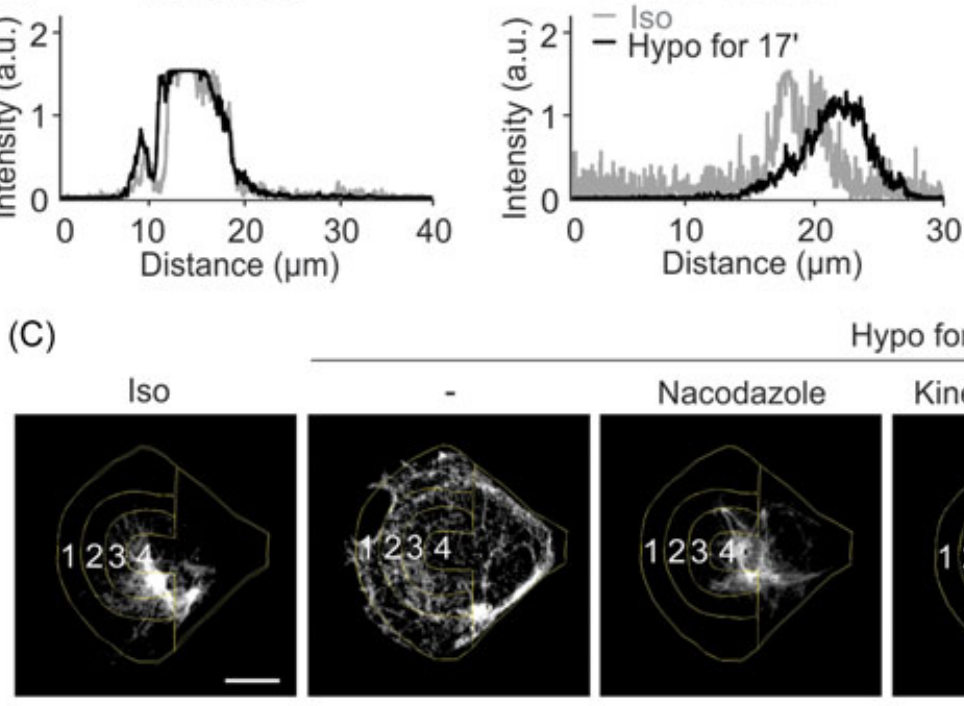

Dynein inh.

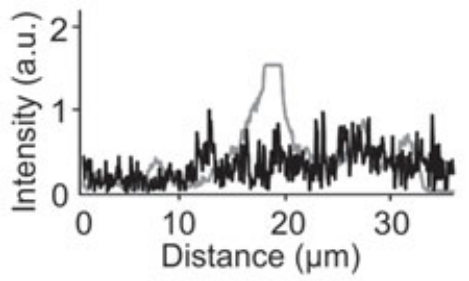

Hypo for $10^{\prime}$
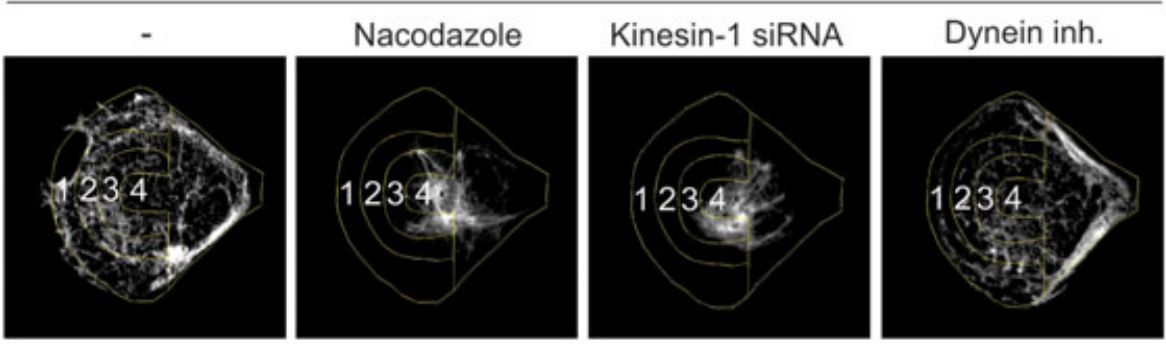

(D)

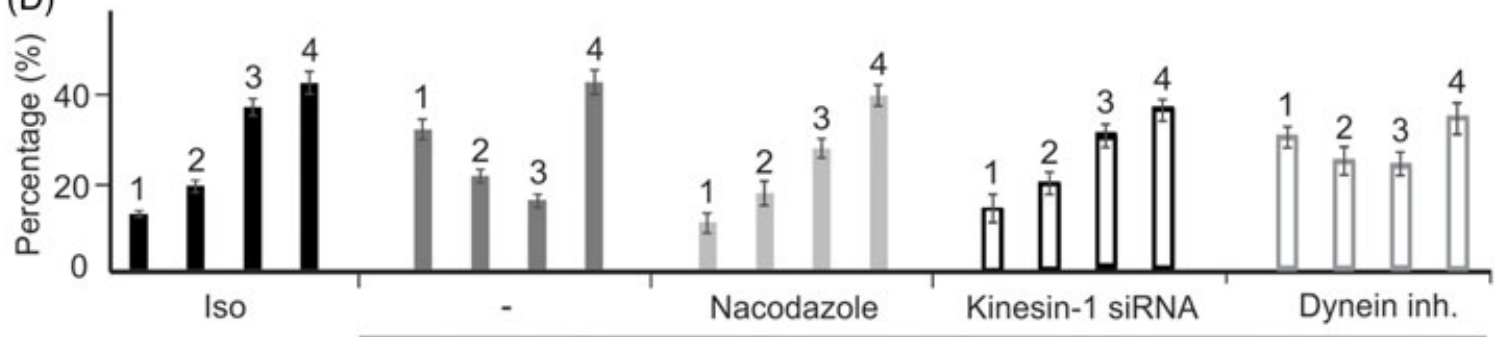

Hypo for $10^{\prime}$

FIGURE 3 Continued. 
(A)

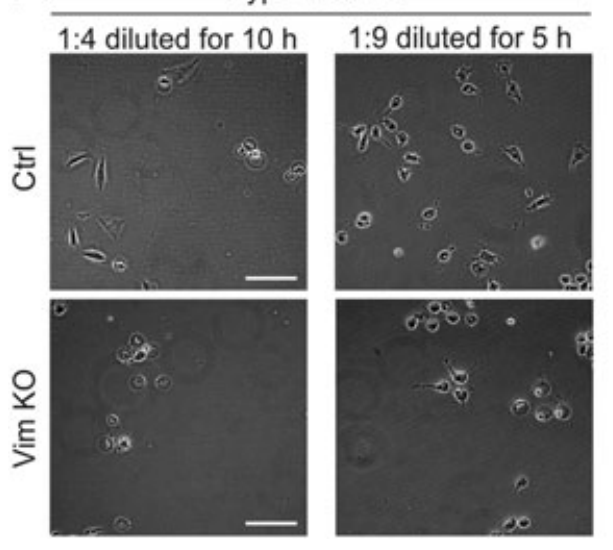

(B)
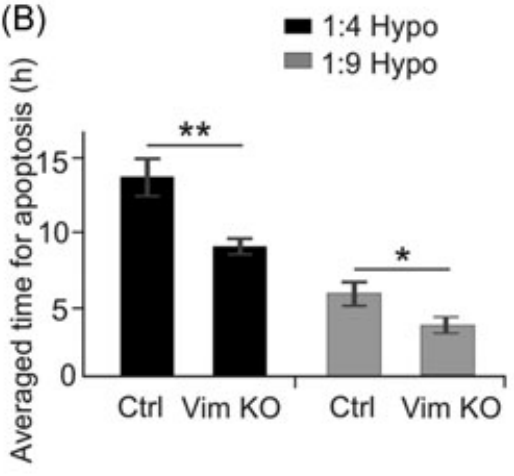

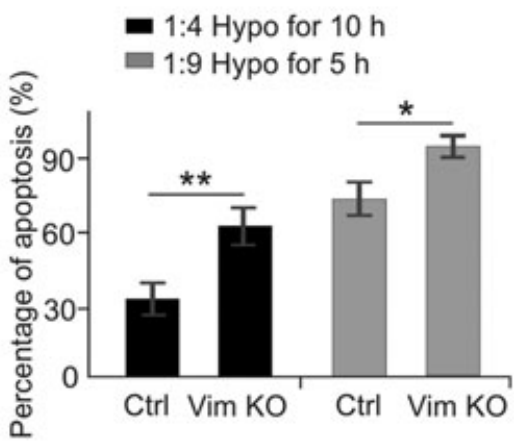

F I G URE 4 Vimentin IFs protect cells from apoptosis induced by hypo-osmotic shock. A, Representative morphogenetic images of control and vimentin KO cells under 1:4 and 1:9 diluted hypo-osmotic medium for 10 hours and 5 hours, respectively. Bars $=100 \mu \mathrm{m}$. B, Quantifications of the average duration and proportion of hypo-osmotic induced apoptosis. ${ }^{* *} P<0.01$ and ${ }^{*} P<0.05$ ( $t$ test). The data are presented as mean \pm SEM. $n=38$ for control in 1:4 diluted, 29 for control in 1:9 diluted, 39 for vimentin KO in 1:4 diluted, and 26 for vimentin $\mathrm{KO}$ in 1:9 diluted hypo-osmotic stress. IF, intermediate filament

observed cell apoptosis in iso- and hypo-osmosic conditions. The hypo-osmotic stress dramatically reduced the averaged time for apoptosis and thus increased the proportion of apoptosis (Figure 4A and 4B), implying the critical role of osmosis in cell survival. Vimentin knockout cells generated in our previous study ${ }^{11}$ showed severe apoptosis rate under both higher and lower hypoosmotic shock (Figure 4A and 4B), indicating the defects in cell survival induced by osmotic stress are systematically regulated, where vimentin IFs play an important protective role.

\section{4 | DISCUSSION}

Osmotic shock is universal stress in multicellular organisms, especially on the epithelial surface or during tumor metastasis. Animals limit osmotic shock by establishing an internal aqueous environment in which intravascular water and electrolytes are subject to sensitive and dynamic organism-based homeostatic regulation. The osmosensory signal transduction network in animals is very extensive and tightly linked to the control of critical physiological functions, such as apoptosis. Whereas the role of the cytoskeleton, especially IFs in hypo-osmotic conditions remain to be established. Our study demonstrated the physiological importance of IFs in hypo-osmotic stress and provided the hints of mechanisms underlying IFs-mediated sensing, and how the IFs are reorganized upon hypotonic stress (Figure 5).

Evidence has accumulated demonstrating that the turnover of IFs network relies on the balance between actin-based retrograde flow and bidirectional microtubulemediated transport. ${ }^{1117,18}$ Depolymerization of microtubules results in the dramatic retraction of the vimentin network to the perinuclear region of the cell. ${ }^{16}$ In retrograde flow, actin transverse arcs are the dominate machinery for IFs population, while the dynein-dependent retrograde transport with faster speed. In anterograde flow, vimentin is mainly dependent on kinesin motor and microtubules. ${ }^{17}$ These different types of motions control the organization of the IFs network in normal conditions. In our study, by using the drug treatment and siRNA methods, we identified that in a pathological condition, for example, osmotic stress, redistribution, and transport of vimentin IFs are guided by the same cytoskeletal structures and molecular motor proteins.

F I G U RE 3 Vimentin redistribution under hypotonic stress is microtubule and kinesin motor dependent. A, Time-lapse imaging of U2OS cells expressing vimentin-mCherry reveal the changes of vimentin under hypo-osmotic shock treated with nocodazole, kinesin-1 siRNA and dynein inhibitor. Yellow dash line indicated the outline of the cell. Bars $=10 \mu \mathrm{m}$. B, Line profiles (indicated by the yellow line in panel A) of vimentin intensity from representative time-lapse imaging showed the distribution change of vimentin upon hypo-osmotic shock. C, Localization of vimentin in hypotonic-cultured cells grown on micropatterns treated with nocodazole, kinesin-1 siRNA and dynein inhibitor. Bars $=10 \mu \mathrm{m}$. D, Quantification of vimentin intensity in four segments upon 10 minutes hypo-osmotic shock. $\mathrm{n}=32 \mathrm{for}$ iso-, 36 for hypo-, 26 for nocodazole treated hypo-, 28 for kinesin-1 siRNA hypo- and 24 for dynein inhibited hypo-osmotic stress. The data are presented as mean \pm SEM. siRNA, small interfering RNA 


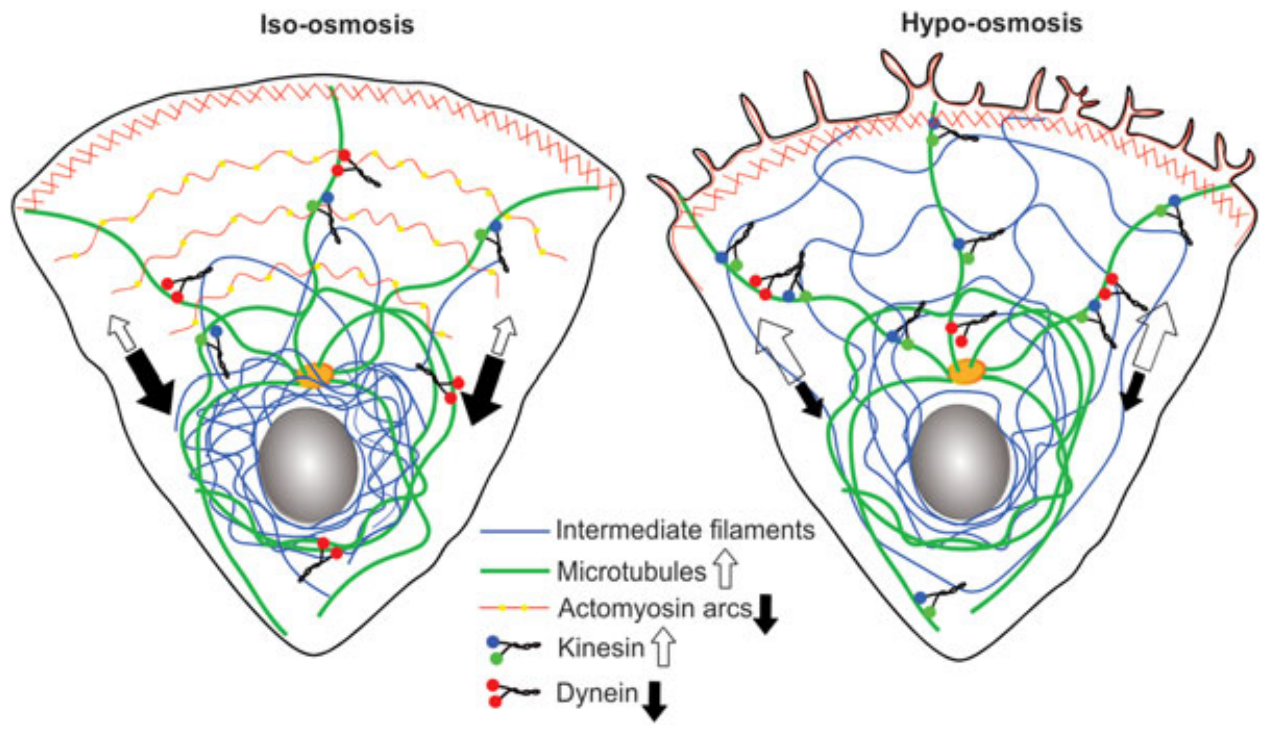

F I G U RE 5 Schematic diagram of vimentin intracellular distribution in iso- and hypo-osmotic culture

There are three potential mechanisms controlling the elongation of the IF network. (1) Guided polymerization along microtubules. ${ }^{19}$ (2) Active transport of pre-existing filaments. ${ }^{20-22}$ (3) Fusion of squiggles. ${ }^{20-22}$ We excluded the first two models and hypothesized that the third theory is maybe most believable, because (1) the soluble (disassembled) compared with insoluble (assembled) fractions of vimentin were increased during extension (Figure 2A and 2B). (2) The endogenous vimentin protein level is not dramatically changed during hypo-osmotic shock (Figure S2E). (3) The total vimentin fluorescence intensity did not fluctuate dramatically within 15 minutes upon hypo-osmotic shock in live-cell imaging (Figure 2C). (4) There are very short filamentous structures were visualized under hypo-osmotic shock (Figure 1E; Figure S2A).

Furthermore, what is the physiological impact of vimentin IFs extension in hypo-osmotic conditions? One possible explanation is that the extension protects the cell from collapse in such dramatic stress, suggesting the structure supporting role of vimentin IFs. Although actin filaments become cortical and generate many protrusions, and microtubules become thinner and diffuse, IFs appear to reconstruct most dramatically under hypo-osmotic shock. The different responses to osmotic stress that we observe in the three cytoskeleton filament systems also point to another functional aspect of IFs in evolution. If microtubules are intrinsically unable to withstand pressure, and filamentous actin needs to be remodeled to accommodate cell swelling, this leaves the IFs network as the only one of the three cytoskeleton filament systems that can be maintained through hypo-osmotic stress, to provide a reference framework for the steady state of the cell, which may be one of the driving forces behind the evolution of the IFs cytoskeleton.

Collectively, our study showed the dramatic spatial change of vimentin and nestin IFs upon the hypoosmotic shock, which depend on microtubules assembly and kinesin level. However, the precise molecular mechanism by which IFs response to osmotic stress and the related cellular function remain important challenges for future research.

\section{ACKNOWLEDGEMENTS}

We thank Pekka Lappalainen (Institute of Biotechnology, University of Helsinki) and Gaoxiang Ge (Shanghai Institute of Biochemistry and Cell Biology, Chinese Academy of Science) for discussions and critical reading of the manuscript. We thank Biological Imaging and Instrumental Analysis Core Facility, Institut Pasteur of Shanghai for imaging technical support and advice on data analysis.

\section{FUNDING INFORMATION}

This study was supported by the "100 talents program" from the Chinese Academy of Sciences, collaborative research grant (Grant no. KLMVI-OP-201904) of CAS Key Laboratory of Molecular Virology \& Immunology, Institut Pasteur of Shanghai, and the starting grant (Grant no. 1185170000) of Institut Pasteur of Shanghai, Chinese Academy of Sciences. F. Cheng would like to thank Sigrid Jusélius foundation, the National Natural Science Foundation of China (Grant no. 81702750) and the Basic Research Project of Shenzhen (Grant no. JCY20170818164756460) for funding. 


\section{CONFLICT OF INTERESTS}

The authors declare that they have no conflict of interests.

\section{ORCID}

Yaming Jiu (10) http://orcid.org/0000-0002-8601-8820

\section{REFERENCES}

1. Miyakawa H, Woo SK, Chen CP, Dahl SC, Handler JS, Kwon HM. Cis- and trans-acting factors regulating transcription of the BGT1 gene in response to hypertonicity. Am J Physiol. 1998;274:F753-F761.

2. Urban JP, Hall AC, Gehl KA. Regulation of matrix synthesis rates by the ionic and osmotic environment of articular chondrocytes. J Cell Physiol. 1993;154:262-270.

3. Zhou YY, Yao JA, Tseng GN. Role of tyrosine kinase activity in cardiac slow delayed rectifier channel modulation by cell swelling. Pflugers Arch. 1997;433:750-757.

4. Finan JD, Guilak F. The effects of osmotic stress on the structure and function of the cell nucleus. $J$ Cell Biochem. 2010;109:460-467.

5. Ly DL, Waheed F, Lodyga M, et al. Hyperosmotic stress regulates the distribution and stability of myocardin-related transcription factor, a key modulator of the cytoskeleton. Am J Physiol Cell Physiol. 2013;304:C115-C127.

6. Ding M, Eliasson C, Betsholtz C, Hamberger A, Pekny M. Altered taurine release following hypotonic stress in astrocytes from mice deficient for GFAP and vimentin. Brain Res Mol Brain Res. 1998;62:77-81.

7. D’Alessandro M, Russell D, Morley SM, Davies AM, Lane EB. Keratin mutations of epidermolysis bullosa simplex alter the kinetics of stress response to osmotic shock. J Cell Sci. 2002;115:4341-4351.

8. Meriane M, Mary S, Comunale F, Vignal E, Fort P, GauthierRouviere C. Cdc42Hs and Rac1 GTPases induce the collapse of the vimentin intermediate filament network. J Biol Chem. 2000;275:33046-33052.

9. Tang DD, Bai Y, Gunst SJ. Silencing of p21-activated kinase attenuates vimentin phosphorylation on Ser-56 and reorientation of the vimentin network during stimulation of smooth muscle cells by 5-hydroxytryptamine. Biochem J. 2005;388:773-783.

10. Sinha B, Köster D, Ruez R, et al. Cells respond to mechanical stress by rapid disassembly of caveolae. Cell. 2011;144:402-413.

11. Jiu Y, Ki JL, Tojkander S, Varjosalo M, Eriksson JE, Lappalainen $\mathrm{P}$. Bidirectional interplay between vimentin intermediate filaments and contractile actin stress fibers. Cell Rep. 2015;11:1511-1518.
12. Schoumacher M, Goldman RD, Louvard D, Vignjevic DM. Actin, microtubules, and vimentin intermediate filaments cooperate for elongation of invadopodia. J Cell Biol. 2010;189:541-556.

13. Correia I, Chu D, Chou YH, Goldman RD, Matsudaira P. Integrating the actin and vimentin cytoskeletons. Adhesiondependent formation of fimbrin-vimentin complexes in macrophages. J Cell Biol. 1999;146:831-842.

14. Huber F, Boire A, López MP, Koenderink GH. Cytoskeletal crosstalk: when three different personalities team up. Curr Opin Cell Biol. 2015;32:39-47.

15. Ball EH, Singer SJ. Association of microtubules and intermediate filaments in normal fibroblasts and its disruption upon transformation by a temperature-sensitive mutant of Rous sarcoma virus. Proc Natl Acad Sci USA. 1981;78:6986-6990.

16. Goldman RD. The role of three cytoplasmic fibers in BHK-21 cell motility. I. Microtubules and the effects of colchicine. J Cell Biol. 1971;51:752-762.

17. Leduc C, Etienne-Manneville S. Regulation of microtubuleassociated motors drives intermediate filament network polarization. J Cell Biol. 2017;216:1689-703.

18. Hookway C, Ding L, Davidson MW, Rappoport JZ, Danuser G, Gelfand VI. Microtubule-dependent transport and dynamics of vimentin intermediate filaments. Mol Biol Cell. 2015;26:1675-1686.

19. Vikstrom KL, Lim SS, Goldman RD, Borisy GG. Steady state dynamics of intermediate filament networks. $J$ Cell Biol. 1992;118:121-129.

20. Prahlad V, Yoon M, Moir RD, Vale RD, Goldman RD. Rapid movements of vimentin on microtubule tracks: kinesindependent assembly of intermediate filament networks. $J$ Cell Biol. 1998;143:159-170.

21. Yoon M, Moir RD, Prahlad V, Goldman RD. Motile properties of vimentin intermediate filament networks in living cells. $J$ Cell Biol. 1998;143:147-157.

22. Helfand BT, Mikami A, Vallee RB, Goldman RD. A requirement for cytoplasmic dynein and dynactin in intermediate filament network assembly and organization. J Cell Biol. 2002;157:795-806.

\section{SUPPORTING INFORMATION}

Additional supporting information may be found online in the Supporting Information section at the end of the article.

How to cite this article: Li J, Gao W, Zhang Y, et al. Engagement of vimentin intermediate filaments in hypotonic stress. $J$ Cell Biochem. 2019;1-9. https://doi.org/10.1002/jcb.28591 